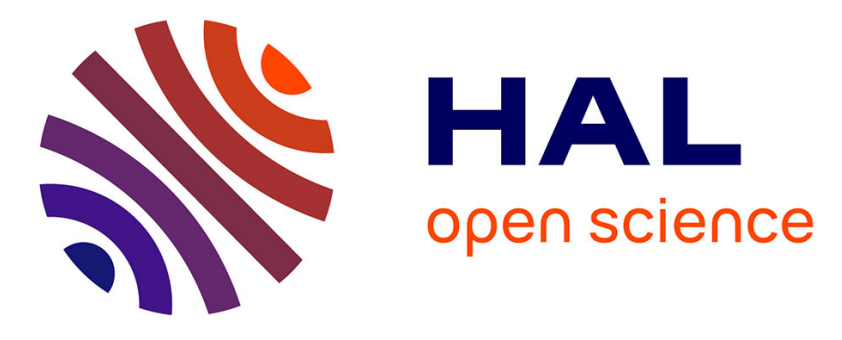

\title{
Hémosidérose rénale consécutive à une hémolyse mécanique: un cas original
}

Julie Zettl, Philippe Mirgaine, Bernadette Aymard, Mathieu Valla, Mohammad Rahmati, Zead Tubail, Fleuria Flechon-Meibody, Benjamin Savenkoff

\section{To cite this version:}

Julie Zettl, Philippe Mirgaine, Bernadette Aymard, Mathieu Valla, Mohammad Rahmati, et al.. Hémosidérose rénale consécutive à une hémolyse mécanique: un cas original. Néphrologie \& Thérapeutique, 2019, 15, pp.115 - 119. 10.1016/j.nephro.2018.11.004 . hal-03486369

\section{HAL Id: hal-03486369 https://hal.science/hal-03486369}

Submitted on 20 Dec 2021

HAL is a multi-disciplinary open access archive for the deposit and dissemination of scientific research documents, whether they are published or not. The documents may come from teaching and research institutions in France or abroad, or from public or private research centers.
L'archive ouverte pluridisciplinaire HAL, est destinée au dépôt et à la diffusion de documents scientifiques de niveau recherche, publiés ou non, émanant des établissements d'enseignement et de recherche français ou étrangers, des laboratoires publics ou privés.

\section{(ㄷ)(1) $\$$}

Distributed under a Creative Commons Attribution - NonCommercial| 4.0 International 
Hémosidérose rénale consécutive à une hémolyse mécanique : un cas original Renal hemosiderosis following mechanical hemolysis: an original case report

Julie Zettl ${ }^{a}$, Philippe Mirgaine ${ }^{a}$, Bernadette Aymard ${ }^{b}$, Mathieu Valla $^{c}$, Mohammad Rahmati $^{\mathrm{d}}$, Zead Tubail ${ }^{\mathrm{a}}$, Fleuria Flechon-Meibody ${ }^{\mathrm{a}}$, Benjamin Savenkoff ${ }^{\mathrm{a}}$

a Service de néphrologie dialyse, CHR de Metz-Thionville, France

${ }^{b}$ Centre de pathologie, 21 Rempart Saint-Thiébault, Metz, France

${ }^{c}$ Service de cardiologie, CHR de Metz-Thionville, France

${ }^{\mathrm{d}}$ Service de chirurgie cardiovasculaire, CHR de Metz-Thionville, France

${ }^{*}$ Auteur correspondant benjamin.savenkoff@gmail.com 


\begin{tabular}{ll} 
Abréviations \\
\hline CGR & concentré de globules rouges \\
DFG & débit de filtration glomérulaire \\
G6PD & Glucose-6-phosphate déshydrogénase \\
HPN & hémoglobinurie paroxystique nocturne \\
HSF & hyalinose segmentaire et focale \\
NTA & nécrose tubulaire aiguë \\
PBR & ponction biopsie rénale \\
PTT & purpura thrombotique thrombocytopénique \\
SHU & syndrome hémolytique et urémique \\
TAVI & Transcatheter Aortic Valve Implantation
\end{tabular}

\section{Introduction}

L'hémosidérose rénale est une cause rare d'insuffisance rénale aiguë, mais également d'insuffisance rénale chronique. Les principales étiologies sont représentées par des hémolyses intravasculaires telles que l'hémoglobinurie paroxystique nocturne $[1,2]$ ou l'hémolyse mécanique sur valve prothétique [3-5], mais il existe bien d'autres cas où l'on peut retrouver cette atteinte. La physiopathologie de l'atteinte rénale implique les molécules d'hème avec leurs mécanismes propres [6-8].

Nous allons présenter le cas original d'une hémolyse mécanique intravasculaire responsable d'une insuffisance rénale aiguë réversible.

\section{Cas clinique}

Un homme âgé de 73 ans se présente aux urgences pour dyspnée et ictère cutanéomuqueux évoluant depuis un mois. II présente comme principaux antécédents :

- une dissection aortique de type A chronique opérée en 2010, avec mise en place d'un tube aortique prothétique dans l'aorte ascendante ;

- une insuffisance aortique modérée chronique ;

- une fibrillation atriale ;

- un flutter ablaté ;

- une insuffisance rénale chronique d'origine vasculaire avec créatininémie à $132 \mu \mathrm{mol} / \mathrm{L}$ (15 mg/L), débit de filtration glomérulaire (DFG) estimé à $45 \mathrm{~mL} / \mathrm{min}$.

Le patient n'était pas connu porteur d'une anémie associée à l'insuffisance rénale jusquelà (taux d'hémoglobine de base à $11,5 \mathrm{~g} / \mathrm{dL}$ ).

Initialement, il présente un tableau de décompensation cardiaque globale associée à un ictère cutanéo-muqueux. L'examen clinique retrouve :

- une dyspnée de stade III ;

- des crépitants bilatéraux;

- un souffle aortique diastolique ;

- des œdèmes des membres inférieurs ;

- une hématurie macroscopique avec des urines rouge porto.

La bandelette urinaire montre $3+$ de sang avec une cytologie urinaire sans hématie. II s'agit donc d'une fausse hématurie qui a posteriori sera expliquée par l'hémoglobinurie présentée par le patient.

La biologie initiale met en évidence une anémie à $9,4 \mathrm{~g} / \mathrm{dL}$, régénérative, hémolytique (haptoglobine effondrée inférieure à $0,1 \mathrm{~g} / \mathrm{L}$, LDH à $1535 \mathrm{UI} / \mathrm{L}$, bilirubine libre à $34 \mathrm{mg} / \mathrm{L}$ ), de type mécanique (schizocytes à $1 \%$ ), une insuffisance rénale stable avec une créatininémie à $121 \mu \mathrm{mol} / \mathrm{L}(13,7 \mathrm{mg} / \mathrm{L})$. II y a une thrombopénie modérée à $100 \mathrm{G} / \mathrm{L}$.

Un bilan d'anémie exhaustif est réalisé en médecine interne, permettant d'éliminer les différentes causes extra-corpusculaires d'anémie hémolytique : test de Coombs négatif, pas de cause infectieuse ou toxique retrouvée... Par ailleurs, les causes corpusculaires d'hémolyse sont également éliminées : pas de clone HPN (hémoglobinurie paroxystique 
nocturne), pas de déficit enzymatique (G6PD, pyruvate-kinase), pas d'argument pour une sphérocytose héréditaire, pas d'hémoglobinopathie héréditaire (électrophorèse de l'hémoglobine normale). Les pièges diagnostiques mimant une anémie hémolytique sont aussi éliminés (pas de carence en vitamine B12 ou en folates, myélogramme normal).

L'échographie transthoracique retrouve des signes de surcharge cardiaque, sans signe de dysfonctionnement du tube aortique. L'insuffisance aortique est stable par rapport aux échocardiographies antérieures.

Un angioscanner aortique est également réalisé, sur lequel la dissection aortique chronique paraît stable et le tube aortique normalement inséré. La cause de cette anémie hémolytique d'allure mécanique reste donc inexpliquée. À noter qu'un purpura thrombotique thrombocytopénique (PTT) est aussi éliminé par un dosage normal de I'ADAMTS-13 (les plaquettes sont modérément abaissées, ce qui rend la probabilité d'un PTT faible).

Le traitement initial en médecine interne et en cardiologie consiste en une déplétion hydrosodée et des transfusions itératives de culots globulaires rouges (dix au total) pour maintenir un taux d'hémoglobine supérieur à $7 \mathrm{~g} / \mathrm{dL}$. À noter que le rendement transfusionnel est très faible, avec une élévation très modeste du taux d'hémoglobine après transfusion.

La réévaluation cardiaque par échocardiographie retrouve une aggravation de l'insuffisance aortique, d'indication chirurgicale par TAVI transventriculaire, la voie classique percutanée étant impossible en raison du tube aortique.

Parallèlement, on assiste à une dégradation de la fonction rénale jusqu'à $353 \mu \mathrm{mol} / \mathrm{L}$ (40 mg/L) de créatininémie, sans critère biologique de dialyse en urgence. II persiste une hématurie macroscopique intense, un ictère cutanéo-muqueux et une anémie hémolytique. Le patient est alors transféré en néphrologie. Une réhydratation prudente est entreprise ainsi qu'un arrêt des traitements diurétiques.

À ce moment de la prise en charge, la principale hypothèse concernant l'insuffisance rénale aiguë est une hémosidérose rénale sur anémie hémolytique mécanique d'origine indéterminée. Les transfusions de CGR sont alors interrompues puisque inefficaces et semblant avoir favorisé l'insuffisance rénale. Nous ne pouvons toutefois pas éliminer formellement un syndrome hémolytique et urémique (SHU), bien qu'il n'y ait pas d'hypertension artérielle associée. À tout cela se rajoutent d'autres causes probables de nécrose tubulaire aiguë (NTA) : l'injection iodée et la déplétion hydrosodée massive pouvant majorer la NTA.

\section{Biopsie rénale}

La biopsie rénale intéresse le cortex rénal et contient 12 glomérules. Les 11 glomérules analysables sont optiquement normaux (Fig. 1). Un seul glomérule est fibreux, en «pain à cacheter ». Les tubes sont de calibre normal, mais il existe quelques cylindres hématiques intraluminaux et le cytoplasme des cellules épithéliales des tubes proximaux contient un pigment brunâtre granuleux, Perls positif, évoquant une hémosidérose importante. L'ensemble de ces lésions tubulaires est compatible avec l'hémoglobinurie (Fig. 2 et 3). II s'y associe une légère artériolosclérose et une artériosclérose modérée. II n'y a pas de dépôts d'amylose, de signe histologique de micro-angiopathie thrombotique ni de lésion de hyalinose segmentaire et focale (HSF). La coloration de Perls est négative au niveau des glomérules. II n'est pas vu de dépôt d'immunoglobuline ou de complément en immunofluorescence directe.

\section{Suivi du patient}

On assiste ensuite à une amélioration spontanée de l'anémie, se stabilisant à $8 \mathrm{~g} / \mathrm{dL}$ d'hémoglobine ainsi qu'une amélioration de la fonction rénale avec une créatininémie stable à $176 \mu \mathrm{mol} / \mathrm{L}(20 \mathrm{mg} / \mathrm{L})$. Nous faisons relire l'angioscanner aortique et il s'avère 
finalement que l'extrémité proximale du tube aortique à la sortie immédiate du ventricule gauche semble en partie désinsérée, avec possible hémolyse mécanique à ce niveau (Fig 4).

Le patient peut finalement être pris en charge en chirurgie cardiaque. En peropératoire, il est effectivement constaté que le tube aortique est désinséré dans sa portion proximale, responsable de l'hémolyse mécanique. Malheureusement, le patient décède en postopératoire d'une défaillance multiviscérale.

\section{Discussion}

II s'agit donc d'un cas original de NTA avec hémosidérose rénale par hémolyse mécanique intravasculaire en rapport avec un tube prothétique aortique désinséré.

Lors d'anémies hémolytiques modérées, il a été démontré que l'atteinte rénale est rare car l'hémoglobine libre est alors complexée par l'haptoglobine dans le compartiment vasculaire et le complexe ainsi formé ne peut pas être filtré par les glomérules, car son poids moléculaire est trop important. En revanche, en cas d'hémolyse massive, l'hémoglobine libre est en excès par rapport à l'haptoglobine, et une partie est alors filtrée par les glomérules et risque donc d'entraîner une toxicité rénale aiguë ou chronique [9], de façon directe par précipitation au niveau tubulaire distal avec l'uromoduline [10], ou bien de façon indirecte, par le biais de dérivés toxiques de l'hémoglobine (fer libre, hème), qui sont responsables de la tubulopathie aiguë ou chronique avec accumulation de ces dérivés dans les cellules tubulaires (hémosidérose rénale).

Le tableau clinique d'hémosidérose rénale aiguë est celui d'une insuffisance rénale aiguë, dans un contexte d'anémie hémolytique, accompagnée d'une fausse hématurie macroscopique à types d'urines rouges porto, qui foncent typiquement à la lumière (hémoglobinurie).

Notons l'intérêt de l'IRM en séquence T2* permettant de quantifier le niveau de surcharge en fer au niveau rénal, notamment chez des patients drépanocytaires ou porteur d'une HPN. Cela se traduit par un hyposignal cortical en T2*, lieu où l'hème se dépose en grande quantité, principalement au niveau tubulaire proximal [11,12].

Le mécanisme physiopathologique implique la molécule d’hème. Aussi appelée porphyrine, elle est constituée d'un ion fer ${ }^{2+}$ et de quatre noyaux pyrroles, chacun composé d'un atome d'azote et de quatre atomes de carbone, ces 4 noyaux étant liés aux sous-unités polypeptidiques qui forment l'hémoglobine (Fig. 5).

Lorsque la concentration en hème est trop importante, elle s'accumule principalement au niveau des cellules tubulaires proximales du cortex rénal du fait de la concentration importante en transporteurs à ce niveau. Cette accumulation toxique est favorisée par des phénomènes ischémiques ou néphrotoxiques (facteurs aggravants). L'hème a un effet toxique direct par perturbation des membranes lipidiques, par activations enzymatiques, par dénaturation de l'ADN, par toxicité mitochondriale et vasoconstriction rénale. L'hème possède également des effets pro-inflammatoires propres tels que l'activation de chémokines ou de leucocytes, engendrant une augmentation de la perméabilité vasculaire $[6,7]$.

On peut signaler le rôle protecteur du système de l'hème oxygénase [6,8], permettant de cataboliser les molécules d'hème en bilirubine, aux propriétés antioxydantes, et ainsi réguler son homéostasie. Ce système est activé en situation de stress pour l'organisme.

À noter que les dépôts de fer libre et d'hème peuvent également entraîner de façon chronique une toxicité glomérulaire, notamment en cas d'hémolyse chronique comme dans les hémoglobinopathies héréditaires. Ainsi, l'équipe de Tenon de Haymann et Stankovic a démontré la toxicité de l'hème dans la génération de lésions de HSF secondaires chez des patients atteints de drépanocytose homozygote SS, avec une corrélation nette entre le degré d'hémolyse et la protéinurie [13]. Des travaux réalisés chez des enfants atteints de thalassémie majeure aboutissent aux mêmes conclusions [13]. À 
noter que chez ces patients, il y a souvent également une hémosidérose secondaire (exhématochromatose secondaire généralisée) à l'hémolyse chronique et aux transfusions itératives, nécessitant alors la prescription de chélateurs du fer.

Les principales causes d'hémosidérose rénale sont les hémolyses intravasculaires, mais également plus rarement les surcharges en fer.

L'hémochromatose génétique est la principale cause de surcharge en fer, mais celui-ci se dépose ordinairement dans d'autres organes tels que le foie, les articulations, le cœur, le pancréas, l'hypophyse... L'atteinte rénale de l'hémochromatose est rare, bien plus souvent attribuée à des dépôts d'IgA secondaires à une atteinte hépatique [14]. Ceci semble démontrer que plus que le fer libre, ce sont bien les dépôts d'hémoglobine et d'hème qui sont réellement néphrotoxiques.

II y a toutefois un cas décrit d'insuffisance rénale aiguë avec glomérulonéphrite rapidement progressive qui est imputé au seul fer libre dans un contexte d'hémochromatose génétique, mais l'imputabilité du fer libre dans les lésions observées n'a pu être démontrée [14].

Parmi les causes d'hémolyse intravasculaire, il faut séparer les anémies hémolytiques par atteintes corpusculaires (maladie du globule rouge) [3], des causes extra-corpusculaires.

Parmi les causes corpusculaires, comme nous l'avons dit, la drépanocytose homozygote SS et les thalassémies sont des causes classiques d'hémolyse chronique, parfois avec retentissement rénal de manière aiguë, mais également chronique [15]. C'est aussi le cas des déficits enzymatiques en glucose-6-phosphate-déshydrogénase ou de la sphérocytose héréditaire (maladie de Minkowski-Chauffard).

L'hémoglobinurie paroxystique nocturne est une cause d'anémie hémolytique acquise responsable d'hémosidérose à ne pas méconnaître, provoquant des atteintes rénales aiguës, mais également chroniques par hémolyse chronique [1,2]. II s'agit d'une maladie clonale de la cellule souche hématopoïétique caractérisée par une mutation somatique acquise, avec défaut d'inhibition de l'activation de la voie alterne du complément au niveau de la membrane plasmique des cellules sanguines, provoquant une hémolyse principalement nocturne.

Parmi les causes extra-corpusculaires, les étiologies sont nombreuses également.

Bien que rares de nos jours, les incompatibilités ABO lors d'erreurs transfusionnelles sont une autre cause d'hémolyse aiguë massive et d'hémosidérose rénale.

Parmi les nombreuses causes infectieuses d'hémosidérose rénale, citons principalement le paludisme, dont la prévalence reste très élevée dans certaines régions du monde. Si lors des accès pernicieux à Plasmodium falciparum, la nécrose tubulaire aiguë est multifactorielle (précipitation d'hématies parasitées par le Plasmodium, hémolyse, vasoconstriction rénale, choc septique...), l'hémosidérose peut aussi être une cause d'insuffisance rénale, notamment dans les formes chroniques de paludisme [16].

$\mathrm{Au}$ niveau cardiovasculaire, les prothèses valvulaires sont souvent responsables d'hémolyses mécaniques a minima [5] et lorsque le phénomène devient plus massif, il est important de suspecter un dysfonctionnement de la prothèse. Ces dysfonctions sont même responsables, dans certains cas, de véritables insuffisances rénales chroniques par hémosidérose rénale $[3,4]$. Historiquement, cette cause était très fréquente en raison d'une durée de vie réduite des prothèses valvulaires mécaniques [17]. Cette situation est heureusement devenue beaucoup plus rare de nos jours.

Dans notre cas clinique, le patient était porteur d'une prothèse au niveau de l'aorte ascendante (tube aortique) du fait d'une dissection aortique de type A. Initialement, les différents examens d'imagerie ne montraient pas d'anomalie patente à ce niveau. Toutefois, compte tenu de la certitude d'hémolyse mécanique sans autre étiologie retrouvée, associée à une hémosidérose rénale histologique, nous avons fait relire le scanner par les radiologues qui ont finalement évoqué une possible désinsertion a minima du tube aortique dans sa portion proximale (Fig. 4). Les chirurgiens cardiovasculaires ont 
finalement tenté de remplacer le tube aortique prothétique désinséré. En peropératoire, le diagnostic de désinsertion proximale du tube aortique a été confirmé.

Par ailleurs, il n'y a pas eu d'incompatibilité transfusionnelle ABO, mais il semble que les transfusions itératives aient aggravé l'hémolyse du patient et l'insuffisance rénale aiguë. En effet, le rendement transfusionnel était très faible et très peu durable, ce qui semblait indiquer une hémolyse mécanique très rapide des hématies transfusées au niveau du tube aortique désinséré.

\section{Conclusion}

La toxicité rénale d'une hémolyse aiguë ou chronique, notamment intravasculaire avec risque de précipitation intratubulaire de l'hémoglobine filtrée (cylindres hématiques) ainsi que le risque de formation de dépôts tubulaires intracellulaires d'hème (hémosidérose rénale), doit donc être connue des néphrologues et suspectée lorsqu'une insuffisance rénale aiguë ou chronique survient dans ce contexte. La PBR confirme le diagnostic, notamment grâce à la coloration de Perls qui permet de révéler les dépôts granuleux de fer ferrique de l'hémosidérine dans le cytoplasme des cellules tubulaires. L'IRM rénale avec pondération T2* est une alternative à la PBR, notamment chez les patients anticoagulés en raison de leur valve pour mettre en évidence cette hémosidérose rénale. II s'agit le plus souvent d'une nécrose tubulaire aiguë, mais l'atteinte rénale peut évoluer vers une néphropathie tubulo-interstitielle chronique, voire vers une glomérulopathie, notamment une HSF secondaire. La prise en charge étiologique de l'hémolyse est la pierre angulaire du traitement de ce type de néphropathie, associée aux mesures de néphroprotection habituelles.

\section{Déclaration d'intérêts}

Les auteurs déclarent n'avoir aucun conflit d'intérêt en lien avec la publication de cet article

\section{Références}

[1] Puri V, Gandhi A, Sharma S. Renal biopsy in paroxysmal nocturnal hemoglobinuria: An insight into the spectrum of morphologic changes. Indian J Nephrol. 2017;27:284-8.

[2] Hussain S, Qureshi A, Kazi J. Renal involvement in paroxysmal nocturnal hemoglobinuria. Nephron Clin Pract. 2013;123:28-35.

[3] Leonardi P, Ruol A. Renal hemosiderosis in the hemolytic anemias: Diagnosis by means of needle biopsy. Blood. 1960;16:1029-38.

[4] Qian Q, Nath KA, Wu Y, Daoud TM, Sethi S. Hemolysis and acute kidney failure. Am J Kidney Dis. 2010;56:780-4.

[5] Somody E, Ferrières J, Dumazer P, Mignon-Conte M, Galinier F, Bernadet P. Renal hemosiderosis caused by chronic hemolysis in a patient with a Saint-Jude mitral valve prosthesis. Arch Mal Cœur Vaiss. 1993;86:111-3.

[6] Tracz MJ, Alam J, Nath KA. Physiology and pathophysiology of heme: Implications for kidney disease. J Am Soc Nephrol. 2017;18:414-20.

[7] Deuel JW, Schaer CA, Boretti FS, Opitz L, Garcia-Rubio I, Baek JH, et al. Hemoglobinuria-related acute kidney injury is driven by intrarenal oxidative reactions triggering a heme toxicity response. Cell Death Dis. 2016;7.

[8] Pittock ST, Nath KA. Induction of heme oxygenase-1 as a protective response against heme protein-induced renal injury. Heme oxygenase in biology and medicine. 2002;24150.

[9] Lathem. The renal excretion of hemoglobin: regulatory mechanisms and the differential excretion of free and protein-bound hemoglobin. J Clin Invest. 1959;38:652. 
[10] Khalighi MA, Henriksen KJ, Chang A, Meehan SM. Intratubular hemoglobin casts in hemolysis-associated acute kidney injury. Am J Kidney Dis. 2015;65:337-41.

[11] Verswijvel G, Vanbeckevoort D, Maes B, Oyen R. Paroxysmal nocturnal haemoglobinuria, MRI of renal cortical haemosiderosis in two patients, including one renal transplant. Nephrol Dial Transplant. 1999;14:1586-9.

[12] Schein A, Enriquez C, Coates T, Wood J. Magnetic resonance detection of kidney iron deposition in sickle cell disease: a marker of chronic hemolysis. J Magn Reson Imaging. 2008;28:698-704.

[13] Haymann JP, Stankovic K, Levy P, Avellino V, Tharaux PL, Letavernier E, et al. Glomerular hyperfiltration in adult sickle cell anemia: a frequent hemolysis associated feature. Clin J Am Soc Nephrol. 2010;5:756-61.

[14] Ozkurt S, Acikalin MF, Temiz G, Akay OM, Soydan M. Renal hemosiderosis and rapidly progressive glomerulonephritis associated with primary hemochromatosis. Renal Failure. 2014;36:814-6.

[15] Calazans LM, De Souza Santos RF, de Souza Gonçalves M, Dos-Santos WL, Rocha PN. Renal hemosiderosis complicating sickle cell anemia. Kidney Int. 2012;8:709.

[16] Kissou SA, Cessouma R, Barro M, Traoré H, Nacro B. Insuffisance rénale aiguë et paludisme à Plasmodium falciparum : à propos d'un cas. Arch Ped. 2011;19:34-7.

[17] Roberts WC, Morrow AG. Renal hemosiderosis in patients with prosthetic aortic valves. Circulation. 1966;33:390.

Figure 1. PBR : les glomérules sont histologiquement normaux (trichrome vert de Masson $x$ 200).

Figure 2. PBR : cylindres hématiques dans la lumière tubulaire (trichrome vert de Masson $x$ 400).

Figure 3. PBR: dépôts granuleux bleutés dans le cytoplasme des cellules tubulaires proximales (Perls $\times 100$ ).

Figure 4. Angioscanner thoracique en coupe axiale: la flèche rouge montre la désinsertion de la partie proximale du tube prothétique dans l'aorte ascendante avec le faux chenal.

Figure 5. Structure chimique de la molécule d'hème. 


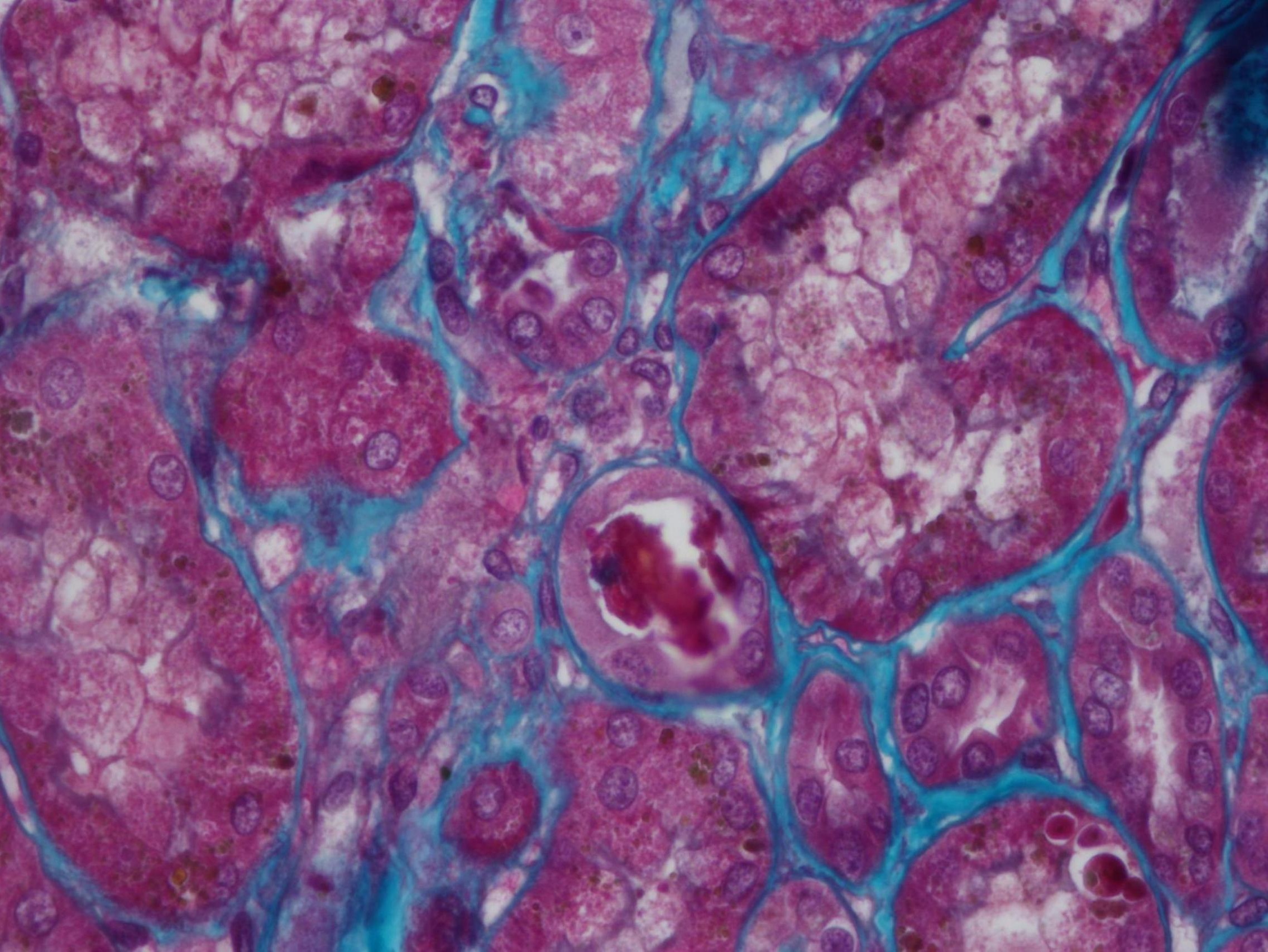




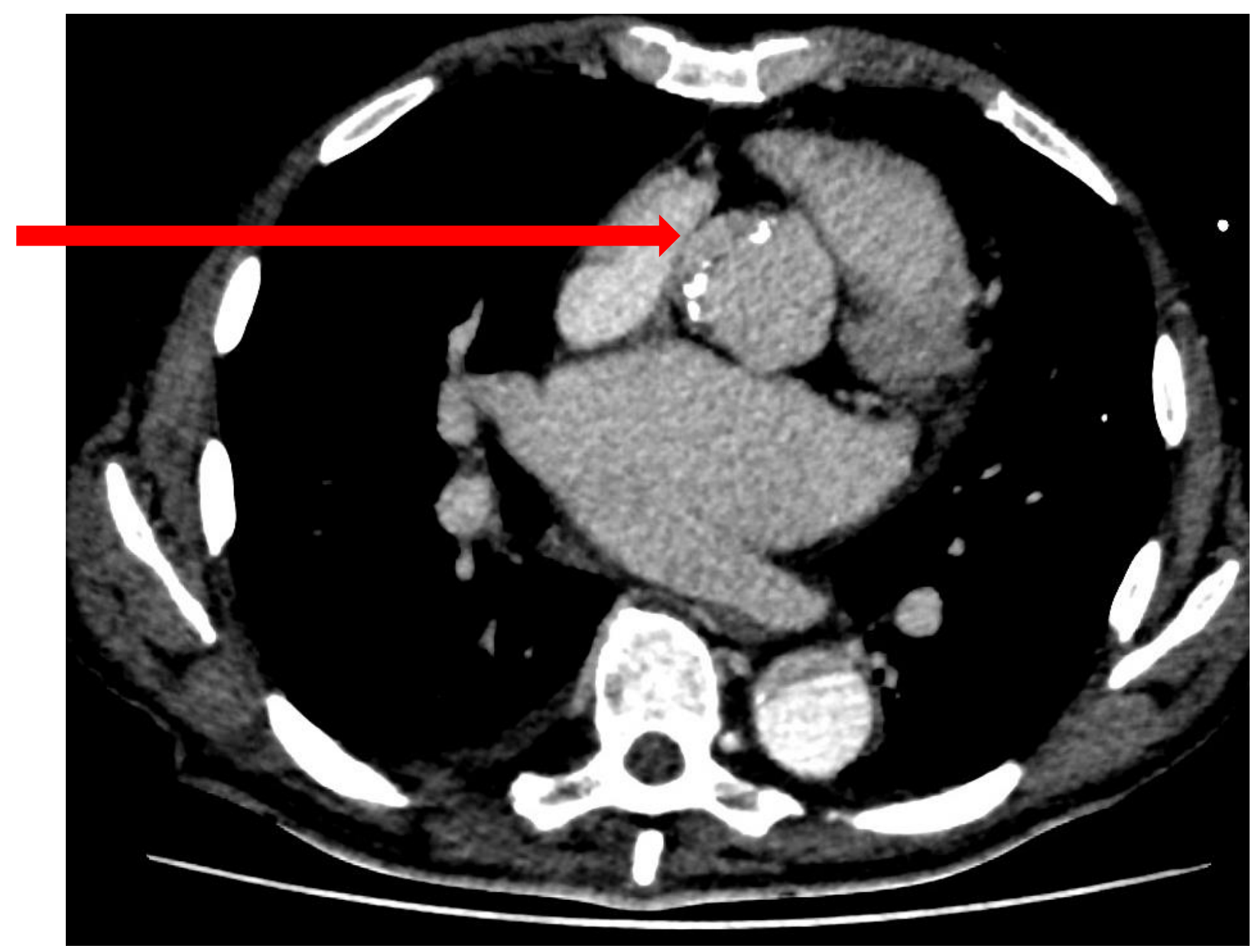


